\title{
Force-Induced Insulin Dimer Dissociation: A Molecular Dynamics Study
}

\author{
Taeho Kim, Alexander Rhee and Christopher M. Yip* \\ Chemical Engineering \& Applied Chemistry, Institute of Biomatrials \& Biomedical Engineering, and The Terrence \\ Donnelly Centre for Cellular and Biomolecular Research, University of Toronto, 160 College St., Toronto, ON, \\ Canada M5S 3E1
}

\section{Materials and Methods}

The insulin crystal structures used in this study were taken from the RCSB Protein Data Bank (native porcine insulin, 4INS). Molecular dynamics (MD) simulations were performed using GROMACS 3.2.1 ${ }^{1,2}$ with the GROMOS-96 ffG43a1 force field ${ }^{3}$, which has been applied previously in non-equilibrium simulations of the forced-extraction of lipid molecules from model membrane bilayers ${ }^{4}$, ion-channel gating ${ }^{5}$, and tension-activation of titin kinase ${ }^{6}$. All systems were subject to periodic boundary conditions with a water box as shown in Figure S1. The dimer was solvated with the SPC water model and $\mathrm{Zn}^{+2}$ ions were added. After energy minimization using a steepest descent method, the system was equilibrated with $50 \mathrm{ps}$ position restraints on the protein atoms to allow the solvent to relax. All systems were run with a 2-fs time step, a non-bonded cutoff of $0.9 \mathrm{~nm}$ for Van der Waals interactions, the Particle-Mesh-Ewald method for long range electrostatic interactions, and LINCS algorithm to constrain covalent bond lengths. The NPT simulations were performed at $300 \mathrm{~K}$ by coupling the protein, water and ions to an external bath $(0.1 \mathrm{ps})$ and the system was equilibrated for $3 \mathrm{~ns}$. For the steered molecular dynamics (SMD) simulations, the dimer molecule was placed in box $6.5 \times 5.18 \times 23 \mathrm{~nm}^{3}$ (59033 atoms, Figure S1) and oriented parallel to the $z$ direction as shown in AFM experiment setup (Figure 1). The $\mathrm{C}_{\alpha}$ atom of Phe B1 in Monomer I was pulled at constant pull rates, ranging from 0.0025 to $0.01 \mathrm{~nm} / \mathrm{ps},(0.0025,0.005,0.075,0.01)$, while the $\mathrm{C}_{\alpha}$ atom of Phe B1 in Monomer II was fixed. The $\mathrm{C}_{\alpha}$ atom in Monomer I was subject to a harmonic spring potential with a spring constant of 168 $\mathrm{kJ} / \mathrm{mol} / \mathrm{nm}^{2}$. Each simulation took about 97 hours per nano-second on a dual $1.0 \mathrm{GHz}$ G4 PowerPC. The SMD simulations of the force spectroscopy experiments were performed using the AFM pull algorithm ${ }^{4}$ in the GROMACS software package. All models and snapshots prepared using VMD?. Hydrogen bonds in Figure 3 were determined based on the cutoffs for the donor-acceptor distance of $3.0 \mathrm{~nm}$ and for the donor-hydrogen-acceptor angle of $60^{\circ} 1,7$.

\section{Rate-dependent force curves}

To investigate whether the insulin dimer dissociation pathways or mechanism were rate-dependent, SMD simulations were performed at various pull rates $(0.0025,0.005,0.075,0.01 \mathrm{~nm} / \mathrm{ps})$. As shown in Figure S2, the rate-dependent force curves illustrated the effect of pull rate on unbinding pathway. The maximum unbinding force decreased with decreasing pull rate and also depended on the dissociation pathway, which are consistent with the previous studies. ${ }^{8}$ Consistent with AFM experiments, these results clearly showed the dimer dissociation occurred near the limit of extensibility of the insulin Bchain.

\section{References}

(1) Berendsen, H. J. C.; van der Spoel, D.; van Drunen, R. Computer Physics Communications 1995, 91, 43-56.

(2) Lindahl, E.; Hess, B.; van der Spoel, D. Journal of Molecular Modeling 2001, 7, 306-317.

(3) van Gunsteren, W. F.; Kruger, P.; Billeter, S. R.; Mark, A. E.; Eising, A. A.; Scott, W. R. P.; Huneberger, P. H.; Tironi, I. G. Biomolecular Simulation: The GROMOS96 manual and user guide; Hochschuleverlag AG an der ETH Zurich: Zurich, 1996.

(4) Marrink, S. J.; Berger, O.; Tieleman, P.; Jahnig, F. Biophys J 1998, 74, 931-943.

(5) Monticelli, L.; Robertson, K. M.; MacCallum, J. L.; Tieleman, D. P. FEBS Lett 2004, 564, 325-332.

(6) Gräter, F.; Shen, J.; Jiang, H.; Gautel, M.; Grubmüller, H. Biophys J 2005, 88, 790-804

(7) Humphrey, W.; Dalke, A.; Schulten, K. Journal of Molecular Graphics 1996, 14, 33-38.

(8) Merkel, R.; Nassoy, P.; Leung, A.; Ritchie, K.; Evans, E. Nature 1999, 397, 50-53. 


\section{Supplementary Figures}

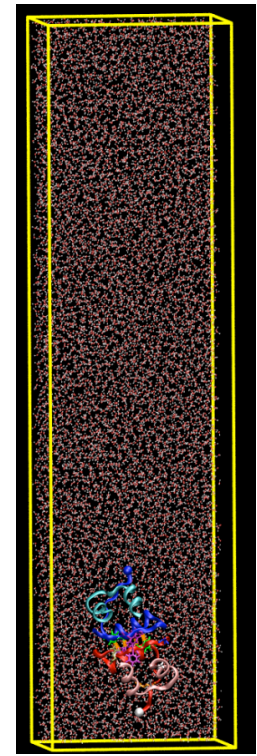

Figure S1. Snapshot of the simulation box. The size of $5.61 \times 4.74 \times 23 \mathrm{~nm}^{3}$ (only part of water molecule are shown for clarity. The $\mathrm{C}_{\alpha}$ (blue) of Monomer I at B1 (A-chain in cyan; B-chain in blue) while Monomer II (A-chain in pink; B-chain in red) remains fixed to the surface via the $\mathrm{C}_{\alpha}$ (white) of Phe B1. Residues involved in specific monomer-monomer interactions are labeled. Hydrogen bonds at inter-monomer interface are shown in orange dotted line.
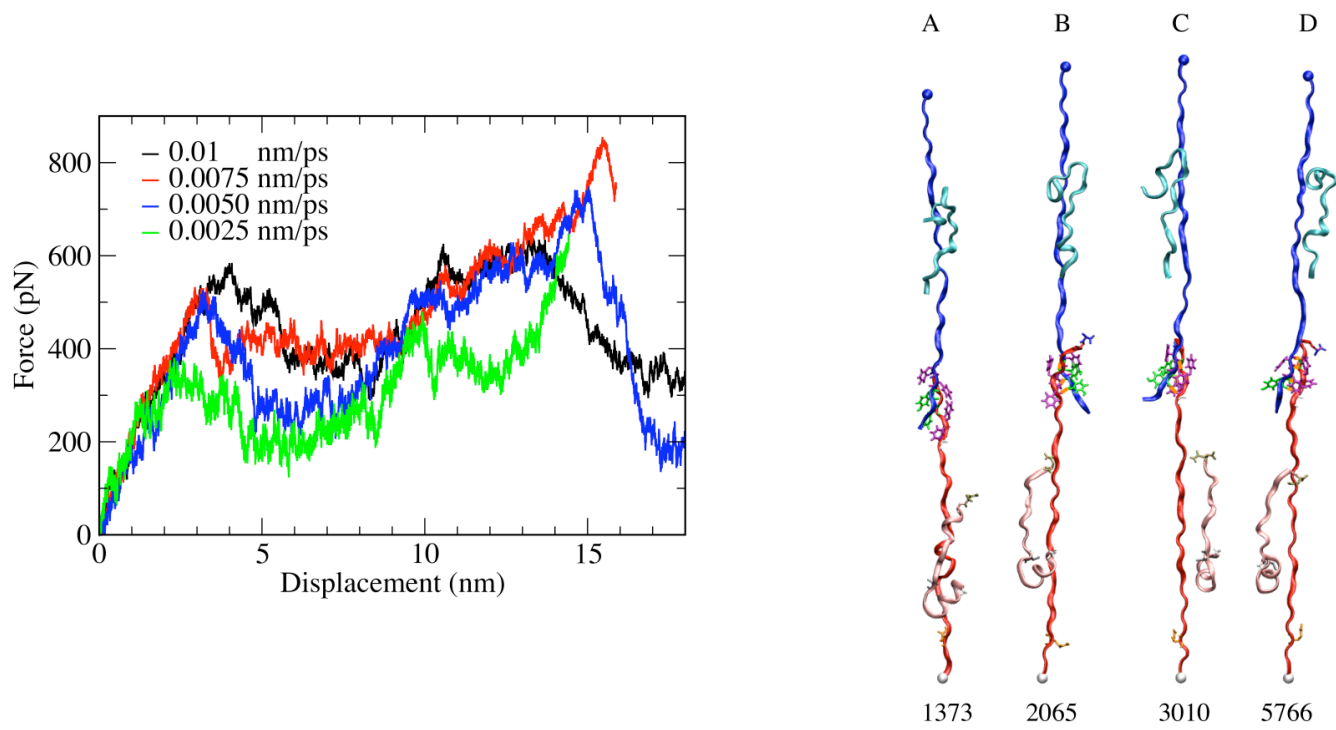

Figure S2. Representative force curves at different pull rates. Plot of force vs. displacement as a function of pull rates at 0.01, 0.075, 0.005, 0.0025 $(\mathrm{nm} / \mathrm{ps})$ during the dissociation processes. Snapshots of the dimer conformation were taken at time $=1373,2065,3010,5766 \mathrm{ps}$ at pull rates of A) 0.01, B) 0.075, C) 0.005, D) $0.0025(\mathrm{~nm} / \mathrm{ps})$, respectively. The displacement reflects the position of the spring. 